\title{
Absenteísmo por causa odontológica: uma revisão de literatura relacionada à ausência no trabalho e à saúde bucal do trabalhador
}

\section{Absenteeism due to dental causes: a literature review regarding absence from work and workers' oral health}

Júnia Nara Gonçalves Mota*

Flávia Godinho Costa Wanderley ${ }^{* *}$

Ricardo Araújo da Silva ${ }^{* * *}$

Tatiana Frederico de Almeida ${ }^{* * * *}$

\section{Resumo}

Introdução: é crescente o interesse dos setores público e privado pelos fatores que acarretam ausências ao trabalho, razão que leva alguns pesquisadores a estudar melhor as relações entre saúde bucal e esse tipo de absenteísmo. Da mesma forma, cada vez mais se observa o interesse das empresas de estender benefícios aos seus trabalhadores, visando à redução do absenteísmo e ao aumento da produtividade. Objetivo: o objetivo deste trabalho é realizar uma revisão de literatura sobre o absenteísmo por causa odontológica, salientando a importância do papel do cirurgião-dentista do trabalho no contexto das políticas públicas no âmbito da saúde do trabalhador. Metodos: o presente artigo caracteriza-se como uma pesquisa de cunho bibliográfico, constituindo uma revisão de literatura referente ao absenteísmo e à saúde bucal do trabalhador. Este trabalho analisou três categorias temáticas, a saber: categoria 1 - doença versus saúde do trabalhador, categoria 2 - saúde bucal do trabalhador, categoria 3 - absenteísmo por causa odontológica. Resultados: verificou-se que o absenteísmo por causas odontológicas traz consequências negativas para qualidade de vida do trabalhador e para as empresas, evidenciando a importância do reconhecimento da especialidade Odontologia do Trabalho. Considerações finais: percebe-se, ao longo dos anos, que embora tenham ocorrido esforços para regulamentar políticas públicas voltadas à saúde bucal, os trabalhadores ainda não são devidamente contemplados com o serviço profissional do cirurgião-dentista do trabalho e sua equipe.

Palavras-chave: Saúde bucal. Absenteísmo. Odontologia.

\section{Introdução}

Atualmente, o trabalho é definido como uma das práticas indispensáveis à vida do ser humano, dele provém a subsistência e a de sua família. No entanto, além de fonte para o sustento, o trabalho deve ser visto como uma atividade prazerosa, na qual o trabalhador sinta realização pessoal e profissional. Assim, fica evidente que aqueles que estão submetidos a condições de trabalho adequadas, aos quais as empresas oferecem programas de assistência à saúde, apresentam rendimento superior àqueles que não recebem esse benefício ${ }^{1}$.

Inserido em um ambiente de trabalho, o ser humano está exposto a diversos fatores que podem influenciar na sua condição de saúde. As ligações Bahia, Brasil.

Mestre em Deontologia e Odontologia Legal pela Universidade de São Paulo, professor assistente de Odontologia, Escola Bahiana de Medicina e Saúde Pública, Salvador, Bahia, Brasil.

**** Doutora em Saúde Coletiva, Universidade Federal da Bahia, professora adjunta de Odontologia, Escola Bahiana de Medicina e Saúde Pública, Salvador, Bahia, Brasil. 
entre trabalho e condições de saúde são consideradas desde a Antiguidade, porém, nem sempre houve a preocupação dos dirigentes de empresas em e atender às necessidades de saúde e bem-estar de seus funcionários. Porém, com o crescente avanço tecnológico e a busca constante por eficiência, empregadores assumiram que a realidade de que seus funcionários faz parte do patrimônio da empresa, e que eles, quando motivados, contribuem para o desenvolvimento e o aumento da produtividade ${ }^{1}$.

Desse modo, as empresas buscam melhorar as condições de trabalho e de atendimento médico-odontológico, visando à redução do absenteísmo e ao aumento da produtividade ${ }^{1}$. O absenteísmo consiste na perda temporária da capacidade de trabalho, determinando sua ausência total ou parcial, interferindo em sua jornada laboral ${ }^{1}$.

Sabe-se que o trabalhador saudável é aquele que detém força de trabalho suficiente para realizar todas as etapas do processo produtivo. Dessa forma, pode-se inferir que a não completude do processo produtivo pelos trabalhadores implica fortemente nos determinantes de saúde-doença, no perfil dos trabalhadores e do trabalho, bem como no quadro epidemiológico e nas práticas de saúde voltadas para o trabalhador ${ }^{2}$.

Em seu ambiente de trabalho, o ser humano está exposto a diversos fatores e agentes que podem causar danos à sua saúde. Assim, consequentemente, suas estruturas bucais estarão sujeitas às alterações que esses fatores podem ocasionar ${ }^{3}$. Não podemos separar as doenças bucais das condições gerais de saúde do ser humano, visto que qualquer problema de origem bucal acarretará em desconforto físico e emocional, prejudicando consideravelmente a saúde geral, gerando influência negativa sobre a capacidade do trabalhador ${ }^{4}$. Midorikawa ${ }^{5}(2001)$ afirma que hoje existem informações de acidentes de trabalho atribuídos a causas básicas de problemas odontológicos.

Dessa forma, o campo da saúde bucal do trabalhador, cujos princípios se aproximam da sua saúde geral, tem como objeto a relação entre saúde bucal e trabalho, tratando de promover, preservar e recuperar a saúde bucal de populações inseridas nos diversos processos de trabalho, contribuindo para a sua qualidade de vida ${ }^{6}$.

Diante da importância da integridade da saúde bucal do trabalhador, o objetivo deste estudo é realizar uma revisão de literatura sobre o absenteísmo relacionado a causas odontológicas, ressaltando a importância da inserção do cirurgião dentista na equipe de saúde e segurança do trabalho.

\section{Metodologia}

O presente trabalho foi desenvolvido a partir de uma pesquisa de cunho bibliográfico, constituindo uma revisão de literatura referente ao absenteísmo e à saúde bucal do trabalhador.
Inicialmente, foi feito um levantamento na literatura em geral, a partir das fontes primárias: livros, leis e portarias federais, textos do Ministério da Saúde, notas técnicas, notícias veiculadas em informativos do Conselho Federal e Regional de Odontologia da Bahia e da Associação Brasileira de Odontologia, relatórios de conferências e encontros regionais sobre saúde do trabalhador.

Como estratégia de busca, foram pesquisados os seguintes descritores: saúde bucal do trabalhador, odontologia e absenteísmo, com os respectivos correspondentes em inglês, tais como oral health worker, dentistry and absenteeism, nas bases de dados da Biblioteca Virtual em Saúde, durante o período de junho a dezembro de 2013. Foram realizados cruzamentos entre dois e três descritores em português e inglês.

Os critérios de inclusão adotados para a seleção dos artigos foram: publicações em português do período de 2002 a 2013, lusofônicos, que abordassem a temática estudada e estivessem disponíveis na íntegra nas bases de dados selecionadas.

Após a etapa de busca e análise das publicações, os achados foram analisados de acordo com três categorias: categoria 1 - doença versus saúde do trabalhador, categoria 2 - saúde bucal do trabalhador, categoria 3 - absenteísmo por causa odontológica.

\section{Revisão de literatura}

\section{Doença versus saúde do trabalhador}

Já encontrada em papiros e outros documentos antigos, a preocupação com a saúde do trabalhador é objeto de estudos desde os primórdios da civilização. Séculos antes de Cristo, no documento Água, ares e lugares, a descrição clínica de intoxicação por saturnismo já era citada, assim como a patologia do trabalho, que foi alvo de preocupações depois da morte de mineiros. Na Idade Média, somente três autores analisaram as relações de trabalho: Agrícola, Paracelso e Bernadino Ramazzini, esse último responsável pelo primeiro estudo ocupacional sistematizado ${ }^{7}$.

Nas últimas décadas, inúmeras modificações no mundo do trabalho influenciam diretamente a saúde individual e coletiva dos trabalhadores. Um conjunto novo e complexo de inovações alterou significativamente a estrutura produtiva, não somente dos países desenvolvidos, mas também dos países de desenvolvimento capitalista tardio, como o Brasil, gerando mudanças na organização, nas condições e relações de trabalho ${ }^{8}$.

Os encargos gerados após a Segunda Guerra Mundial, oriundos da perda de vidas gerada por acidentes de trabalho e por doenças ocupacionais, começaram a ser sentidos pelos empregadores e pelas companhias de seguros. Simultaneamente, a tecnologia industrial e o desenvolvimento de novos 
processos levaram ao surgimento de novas doenças profissionais, desafiando a medicina do trabalho ${ }^{9}$.

No Brasil, essa preocupação surgiu com a chegada da febre amarela em Pernambuco e na Bahia, que acabou dizimando a mão de obra dos engenhos de açúcar e trouxe grandes prejuízos à economia. Até o século XIX, a mão de obra era escrava. Somente após a Revolução Industrial, diante das precárias condições de trabalho, viu-se a necessidade de dispor de serviços de saúde ao trabalhador, visando sobrevivência e produtividade do processo industrial $^{10}$.

Dessa forma, a saúde ocupacional surgiu e implantou serviços de assistência nos locais de trabalho, culminando, anos depois, com a concepção de saúde do trabalhador, que se baseia em um conjunto de práticas em que o trabalhador busca o controle sobre as condições e os ambientes de trabalho, tornando-os saudáveis ${ }^{11}$.

A saúde do trabalhador compreende as relações entre o processo saúde-doença e o trabalho. Sendo assim, trabalhador será todo o indivíduo que exerce uma atividade laboral no mercado formal ou informal, familiar ou doméstico. Doenças ocupacionais são aquelas que podem ser causadas ou agravadas por fatores de risco presentes no local de trabalho: agentes físicos (ruídos, calor, vibração, ventilação, luminosidade, umidade, radiação), químicos (gases, fumo, névoa, neblina e poeira), biológicos (bactérias, fungos, parasitas e vírus) ou até a organização do trabalho (estresse, divisão organizacional, produtividade, repetitividade, jornadas extensas, esforços e posições inadequadas) ${ }^{11}$.

Benedicto et al. ${ }^{12}$ (2010) mencionam um estudo que relata a dificuldade de indivíduos portadores de lesões por esforço repetitivo em realizar uma correta escovação dental, justificando o aumento de problemas bucais em tais portadores. Sendo assim, é importante que a equipe de saúde bucal identifique esses indivíduos a fim de realizar atividades educativas e preventivas com esse grupo.

No Brasil, a lei que determina as diretrizes das ações e serviços de saúde no território nacional, inclusive com relação à saúde do trabalhador, é a Lei Orgânica da Saúde, Lei no 8.080, de 19 de setembro de 1990, que afirma que a saúde é um direito fundamental do indivíduo, e, portanto, o Estado deve prover condições ao seu pleno exercício, certificando acesso universal e igualitário às ações e aos serviços para sua promoção, proteção e recuperação. São fatores determinantes da saúde: a alimentação, a moradia, o saneamento básico, o meio ambiente, o trabalho, a renda, a educação, o transporte, o lazer, bem como o acesso aos serviços essenciais, garantindo às pessoas condições de estabelecer e manter o bem-estar físico, mental e social ${ }^{13}$.

Em 1998, o Ministério da Saúde publicou as portarias $\mathrm{n}^{\circ} 3.120$ e $\mathrm{n}^{\circ} 3.908$, tratando dos procedimentos básicos para a vigilância em saúde do trabalhador e da prestação de serviços nesta área ${ }^{14}$. A publicação da Lista de doenças relacionadas ao trabalho, conforme Portaria oํ 1.339, de 1999, também foi outro avanço ${ }^{15}$.

A saúde do trabalhador é uma área que tem como princípio o desenvolvimento de ações que visem atuar no processo saúde-trabalho-doença, eliminando ou controlando os fatores de risco e danos $^{16}$. Certamente, os profissionais da saúde e os trabalhadores contribuem para a construção dessa área ${ }^{9}$.

\section{A saúde bucal do trabalhador}

A boca, em virtude de sua localização e de suas funções, é uma zona de absorção, retenção e excreção de substâncias tóxicas, sujeita a agressões físicas e mecânicas, além de ser porta de entrada do sistema estomatognático e participar como auxiliar na respiração $0^{3}$.

O conceito de saúde bucal sofreu diversas modificações nos últimos anos. Até os anos 1960, sinônimo de boa saúde era a extração dentária. No regime militar, nos anos 1970, a bandeira foi a odontologia curativa. Naquele período, saúde bucal era o dente estar obturado, não se pensava em prevenção ${ }^{17}$.

É inegável a importância da saúde bucal para o alcance da qualidade de vida, e por isso os indicadores no Brasil são preocupantes: alta experiência de cárie, significativo número de necessidade de uso de próteses e comprometimento periodontal severo ${ }^{18}$.

Dentro do conceito atual de saúde integral, a saúde bucal do trabalhador passa a ser:

[...] parte da atenção à saúde do trabalhador, que trata de promover, preservar e recuperar a saúde bucal do trabalhador, consequente dos agravos, afecções ou doenças do exercício profissional, e que tem manifestações bucais, devendo ter sua ação voltada à prevenção de todos os agravos laborais, ou seja, objetiva a prevenção de doenças decorrentes da atuação profissional e dos acidentes de trabalho ${ }^{19}$.

Pizzato e Garbin ${ }^{2}$ (2006) verificaram que as doenças ocupacionais oriundas da exposição a fatores de risco de origem física, química e biológica podem ter manifestados na cavidade bucal seus primeiros sinais e sintomas.

Existem relatos de estudos relacionando a exposição ocupacional e o aumento da incidência de doenças bucais. A relação entre doenças bucais e o trabalho nas indústrias produtoras de cimento, na área petroquímica, na agricultura e na indústria alimentícia são alguns exemplos ${ }^{20}$. Os agentes químicos, orgânicos ou inorgânicos, são predominantes nos estudos, tanto de revisão quanto empíricos, e são considerados os principais responsáveis por alterações bucais de origem ocupacional, como lesões da mucosa oral, doença periodontal, alterações salivares bem como alguns sintomas orais de dor, xerostomia, ardor e outros ${ }^{21}$.

A dor exerce um importante impacto na qualidade de vida dos indivíduos devido ao sofrimento e 
às limitações que causa. Entretanto, pouco se sabe a respeito da prevalência da dor orofacial e seu impacto no cotidiano de trabalhadores no Brasil ${ }^{22}$.

Vianna e Santana ${ }^{3}$ (2001) associaram efeitos bucais decorrentes de exposições ocupacionais a névoas ácidas e perda mineral da estrutura dental, notando-se maior acometimento em dentes anteriores, o que destaca a necessidade de inserção dos profissionais de odontologia nas equipes de saúde e segurança do trabalhador e higiene industrial.

De Mello $^{23}$ (2006) realizou uma síntese dos principais agentes de risco ocupacional e suas manifestações bucais relacionadas com algumas atividades laborativas. Entre esses agentes, destacaram-se as substâncias químicas utilizadas em processos industriais.

Dividida em dois grandes blocos, a Portaria $\mathrm{n}^{\mathrm{o}}$ 1.339 , de 18 de novembro de $1999^{14}$, retrata de forma fiel a classificação das doenças que remetem à cavidade bucal de diversas profissões. $\mathrm{O}$ primeiro bloco exibe a relação de agentes ou fatores de risco de natureza ocupacional, com as respectivas doenças que podem estar a eles relacionadas. No segundo bloco, apresenta-se a relação de doenças e agentes causais relacionados com o trabalho.

Segundo Mazzili'i (2007), as normas regulamentadoras (NRs) são de observância obrigatória pelas empresas privadas e públicas, assim como pelos órgãos públicos da administração direta e indireta e pelos órgãos dos poderes Legislativo e Judiciário. Essas normas surgiram na Consolidação das Leis do Trabalho e relacionam-se à segurança e à medicina do trabalho.

A Norma Regulamentadora $\mathrm{n}^{-}$4, que trata do Serviço Especializado em Engenharia de Segurança e em Medicina do Trabalho, demonstra a necessidade da presença do profissional de odontologia no setor de saúde do trabalhador, pois os demais profissionais que integram a equipe, como médico do trabalho, engenheiro de segurança do trabalho, enfermeiro do trabalho, técnico em segurança do trabalho e técnico de enfermagem do trabalho, não podem intervir na área de competência do dentista, como prevenir, diagnosticar e tratar afecções próprias da boca ${ }^{24}$.

A NR nำ faz considerações sobre os equipamentos de proteção individual, que também poderiam ser indicados pelo dentista do trabalho, como protetores labiais, protetores bucais e máscaras para respiradores bucais ${ }^{24}$.

A NR no 9 descreve e regulamenta o objetivo e o campo da aplicação dos conhecimentos do Programa de Prevenção de Riscos Ambientais, determinando aplicações de conhecimento específico do dentista do trabalho, como exposições a agentes ${ }^{24}$.

A NR nº 21 estabelece observação por odontologista do trabalho quando houver exposição do trabalhador ao frio e calor excessivos, sobretudo à radiação ultravioleta, que pode levar ao aparecimento de lesões no lábio inferior, nariz e terço médio da face $^{24}$.

A NR n 32 estabelece as diretrizes para implementação de medida de proteção à segurança e à saúde dos trabalhadores em serviços de saúde e afins, e, também, abre espaço para a atuação do dentista do trabalho ${ }^{24}$.

A NR-7 objetiva promover e preservar a saúde dos trabalhadores de uma empresa, devendo elaborar um Programa de Controle Médico de Saúde Ocupacional, independente do número de trabalhadores e do grau de risco da empresa. Cabe ao médico do trabalho coordenar esse trabalho, mas existem ações que são específicas do dentista do trabalho, como atestado em saúde ocupacional odontológico, exames admissionais e periódicos, de retorno ao trabalho e de mudança de função $0^{23}$.

O Projeto de Lei $\mathrm{n}^{\mathrm{0}} 422 / 2007$ alterou o art. 162, secção III, e o art. 68, secção V, do Título II da Consolidação das Leis do Trabalho (Decreto-Lei $\mathrm{n}^{\circ}$ 5.452/1943), obrigando as empresas a manter serviços especializados em segurança, medicina e odontologia do trabalho. Assim, a participação do dentista na equipe de saúde do trabalhador, bem como na realização dos exames admissionais e pré-admissionais, dentre outros, passou a ser obrigatória.

\section{Absenteísmo por causas odontológicas}

Midorikawa $^{25}$ (2000) investigou a odontologia na saúde do trabalhador e apontou dois tipos de absenteísmo: o absenteísmo pela falta ao trabalho e o absenteísmo de corpo presente. O primeiro (absenteísmo tipo I) consiste na falta pura e simples do empregado ao trabalho, levando à perda de produção das horas não trabalhadas, portanto, mais fácil de ser mensurado. Já o segundo absenteísmo (tipo II) ocorre quando o trabalhador apresenta algum problema de saúde que impede seu total desempenho e produtividade, mesmo não faltando ao trabalho.

Conforme Mazzilli²4 (2007), o absenteísmo tipo II é responsável pelas principais causas de falta de produtividade nas empresas públicas e privadas, além de ser um importante fator de risco para acidentes de trabalho. Na economia competitiva atual, o interesse pelo absenteísmo é crescente, visto que quanto menor sua incidência, maior será a rentabilidade e o crescimento sustentável. Disfunções bucais podem ter consequências diretas sobre a produtividade dos trabalhadores, pois muitas doenças sistêmicas têm origem em problemas bucais.

Miller $^{26}$ (1978), avaliando o efeito das doenças bucais na qualidade de vida da população, observou maior prevalência de absenteísmo por causa odontológica em pessoas do gênero feminino. Midorikawa $^{25}$ (2000) afirmou que trabalhadores com boas condições de saúde bucal tornam-se mais desinibidos, extrovertidos e sociáveis. Enquanto que o trabalhador que possui problemas odontológicos 
manifesta vários sinais e sintomas, como dor, desconforto, sentimento de inferioridade e estresse.

Hooper $^{27}$ (1942) estudou o absenteísmo em indústrias norte-americanas e afirmou que, pelo menos, 25\% do absenteísmo por doenças não ocupacionais se deve às condições bucais. Mencionou, também, a relação entre infecções orais e doenças sistêmicas, discutindo a importância da profilaxia como prevenção, e que a preocupação com os serviços odontológicos deve caber à empresa, ao médico do trabalho, ao cirurgião-dentista e ao próprio trabalhador.

Almeida e Vianna ${ }^{28}$ (2005) afirmaram que, no campo da saúde bucal coletiva, as iniciativas até aquele momento voltavam-se quase que exclusivamente para a parcela da população escolar. No entanto, mudanças no perfil demográfico e epidemiológico da população inferem na necessidade da atenção aos diferentes grupos etários. Nesse contexto, é de extrema importância a observação da população economicamente ativa, que está exposta não somente aos fatores etiológicos das principais doenças bucais, mas, também, aos fatores de risco relacionados aos acidentes de trabalho.

Duffy $^{29}$ (1996), estudando a incidência de faltas devido a problemas odontológicos na Shell (Royal Dutch Shell, indústria de petróleo anglo-holandesa), no período de 1988 a 1994, verificou que o absenteísmo por causas odontológicas gerava um grave problema para a empresa e que a implantação de programas de promoção de saúde bucal, educação em saúde bucal, tratamento de afecções e exames periódicos poderiam alterar essa situação.

Alterações de ordem psicológica, como falta de concentração, que provocam acidentes e erros técnicos, ou alterações de humor, que geram discussão e intolerância, são exemplos de situações ligadas à dor de dente, que podem ser prevenidas e minimizadas com a implantação da odontologia do trabalho nas empresas ${ }^{30}$.

\section{Discussão}

O equilíbrio entre a saúde do trabalhador e a produtividade é rompido pela falta no trabalho relacionada à doença. $\mathrm{O}$ absenteísmo compreende dois episódios distintos: a) o absenteísmo por motivo de doença, aquele relacionado às ausências provenientes de problemas da própria saúde ou de dependentes, englobando procura por diagnóstico, terapia e assistência, não sendo necessária a interrupção do exercício laborativo; b) o absenteísmo-doença, que se dá pela incapacidade de execução das atividades devido a alguma doença ou lesão acidental, sendo necessária a ausência.

De acordo com Peres et al. ${ }^{1}$ (2006), indicadores de prevalência e incidência do absenteísmo por causas odontológicas, relacionando faixa etária, gênero, tempo médio de afastamento, principais proble- mas odontológicos, além dos prejuízos acarretados à economia, são relatados por alguns autores.

Diferentemente do absenteísmo tipo I, que ocorre quando o empregado se ausenta temporariamente do trabalho, no absenteísmo tipo II, também chamado de presenteísmo, o trabalhador se encontra presente fisicamente, porém mental e emocionalmente distante, causando prejuízos e descontentamento à empresa e aos clientes. Dores de cabeça, musculares, nas costas, insônia e depressão são alguns dos sintomas mais comuns do presenteísmo, porém, o mais frequente é o estresse ${ }^{24,25}$.

Condições bucais precárias podem acarretar absenteísmo e declínio na produtividade em uma empresa. Alterações de humor e comportamento são notadas naqueles trabalhadores que sofrem, por exemplo, com dor de dente. Além de estar mais propícios a erros e acidentes de trabalho, as relações interpessoais também podem ser prejudicadas por irritação e intolerância.

A maioria dos autores pesquisados considera o absenteísmo de corpo presente como algo corriqueiro, principalmente aquele relacionado às causas odontológicas, porém, é uma situação de difícil mensuração. Dessa forma, o presente trabalho quer salientar a necessidade de programas, estudos e ações que visem à redução desse problema.

Também é unânime entre os autores a importância da prevenção e que a preocupação com o serviço odontológico cabe à empresa, ao cirurgião-dentista e ao próprio trabalhador, tendo sua eficácia melhorada quando a oferta do serviço é integrada ao programa de saúde da empresa.

Reconhecida pelo Conselho Federal de Odontologia em 2001, por meio da Resolução no 22, de 27 de dezembro, a odontologia do trabalho é uma especialidade recente. A Resolução no 25, de 2002, definiu as áreas de competências para atuação do dentista do trabalho. A especialidade foi incluída pelo Ministério do Trabalho e Emprego na Classificação Brasileira de Ocupações, recebendo o código 2232-76, podendo ser identificada também como odontologia ocupacional ${ }^{23}$.

A partir dessa resolução, as competências do especialista em odontologia do trabalho dentro das empresas incluem: identificação dos fatores ambientais de risco à saúde bucal, verificação da obediência das medidas de segurança e higiene, planejamento e promoção de atividades de educação em saúde bucal, investigação dos índices de mortalidade e morbidade com causa bucal, realização de exames odontológicos admissionais.

No contexto de valorização dos recursos humanos, a odontologia do trabalho ganha ênfase, com o objetivo de estudar, interpretar e solucionar os diferentes problemas bucais que atingem os trabalhadore ${ }^{16}$. Em um mundo de constantes mudanças, economia globalizada e países emergentes, a odontologia do trabalho traz um campo de atuação bas- 
tante promissor para o cirurgião-dentista, exigindo qualificação diferenciada ${ }^{24}$.

Entre as NRs, existem algumas em que os conhecimentos do dentista do trabalho poderiam somar, aumentando a efetividade dos serviços de saúde e segurança do trabalhador. Porém, apesar de tantas NRs, a prática odontológica tem sido fundamentada na intervenção cirúrgico-restauradora, mesmo que se saiba que não é possível controlar as doenças bucais dessa forma ${ }^{23}$.

Um dos primeiros passos para a inserção da odontologia na equipe de saúde e segurança do rabalho foi a aprovação do PL no 422/2007.

Os estudos analisados nesta revisão são unânimes em ressaltar a importância da inserção do cirurgião-dentista do trabalho, que, juntamente com os outros profissionais da equipe de saúde do trabalhador, terão condições de fazer um amplo planejamento das atividades de promoção e prevenção de saúde bucal, por meio de um programa de saúde bucal, visando à saúde integral do trabalhador, auxiliando na adoção de medidas que visem, além da completa saúde e bem-estar, o aumento da autoestima e da produtividade e a diminuição do absenteísmo.

\section{Considerações finais}

No Brasil, apesar de terem ocorrido esforços para regulamentar políticas públicas voltadas para a saúde bucal do trabalhador, elas não foram devidamente contempladas com a atuação profissional do cirurgião-dentista do trabalho e sua equipe. Embora já sejam catalogados alguns agentes etiológicos de origem ocupacional, que são responsáveis por agravos à cavidade bucal, existe uma fragilidade na classificação de doenças exclusivas da cavidade bucal, visto que são escassos os estudos correlacionando-os.

É de extrema importância a inserção do cirurgião-dentista do trabalho nas empresas para compor a equipe de segurança e saúde do trabalhador, cooperando com os demais profissionais em todas as ações que visem preservar a integridade social e a manutenção da saúde geral do trabalhador, melhorando sua produtividade e reduzindo o absenteísmo por causa odontológica, problema que interfere diretamente na qualidade de vida e no trabalho do indivíduo.

\section{Abstract}

Introduction: Public and private sectors have been increasingly interested in factors that cause absence from work, which has led some researchers to further study the relationship between oral health and this type of absenteeism, as companies are also gradually more interested in extending benefits to their employees in order to reduce absenteeism and increase productivity. $\mathrm{Ob}$ - jective: To perform a literature review on absenteeism due to dental causes, emphasizing the importance of the role of the occupational dentist in the context of public policies, within the scope of Workers' Health. Methodology: Bibliographical research consisting of a literature review regarding absenteeism and workers' oral health. The present work analyzed three theme categories: Category 1 - Disease vs. Workers' Health, Category 2 - Workers' Oral Health, Category 3 - Absenteeism due to dental causes. Results: It was found that absenteeism due to dental causes presents negative consequences for the quality of life of workers and for the companies, highlighting the importance of acknowledging the expertise of Occupational Dentistry. Final Thoughts: It is noticed, over the years, that although there were efforts to regulate public policies for oral health, it has not been properly covered concerning workers and the professional performance of occupational dentists and their team.

Keywords: Workers. Oral health. Absenteeism. Dentistry.

\section{Referências}

1. Peres SHCS, Theodoro DS, Ribeiro DA, Avila ED, Greghi GA, Silva RPR. Odontologia do trabalho: doenças e lesões na prática profissional. Rev Odontol Araçatuba 2006; 27(1):54-8.

2. Pizzato E, Garbin CAS. Odontologia do trabalho: implantação da atenção de saúde bucal do trabalhador. Odontol Clin Cient 2006; 5(2):99-102.

3. Vianna MIP, Santana VS. Exposição ocupacional a névoas ácidas e alterações bucais: uma revisão. Cad Saude Publica 2001; 17(6):1335-44.

4. Silveira GS. Absenteísmo de causa odontológica 2008 [Trabalho de Conclusão de Curso]. Rio de Janeiro: Escola de Saúde do Exército; 2008.

5. Midorikawa ET. Cuidados com a saúde bucal no ambiente de trabalho. Rev CIPA 2001; 22(257):64-9.

6. Elias MA, Navarro VL. A relação entre o trabalho, a saúde e as condições de vida: negatividade e positividade no trabalho das profissionais de enfermagem de um hospital escola. Rev Lat Am Enfermagem 2006; 14(4):517-25.

7. Minayo-Gomez C, Thedim-Costa SMF. A construção do campo da saúde do trabalhador: percurso e dilemas. Cad Saude Publica 1997; 13(2):21-32.

8. Mendes R, Dias EC. Da medicina do trabalho à saúde do trabalhador. Rev Saude Publica 1991; 25(5):341-9.

9. Oliveira MHB, Vasconcellos LCF. As políticas públicas brasileiras de saúde do trabalhador: tempos de avaliação. Saude em Debate 2000; 24(55):92-103.

10. Brasil. Ministério da Saúde. Doenças relacionadas ao trabalho: manual de procedimentos para os serviços de saúde. Brasília; 2001.

11. Brasil. Lei oㅜ 8.080, de 19 de setembro de 1990. Diário Oficial da União, Brasil, Brasília, 20 de setembro de 1990, seção 1, p. 18055-9.

12. Benedicto EN, Margreiter S, Brando TM, Daruge Junior E, Paranhos LR. Consideração sobre as doenças bucais que afetam os trabalhadores e suas implicações éticas e legais. Perspect Oral 2010; 2(2):51-6.

13. Brasil. Portaria no 3.908 , de 30 de outubro de 1998. Diário Oficial da República Federativa do Brasil, Brasília, 10 de novembro de 1998: seção 1, p. 223-5. 
14. Brasil. Portaria no 1.339 , de 18 de novembro de 1999. Diário Oficial da República Federativa do Brasil, Brasília, 19 de novembro de 1999, seção 1, p. 21.

15. Carvalho ES, Hortense SR, Rodrigues LMV, Bastos JRM, Peres AS. Prevenção, promoção e recuperação da saúde bucal do trabalhador. RGO 2009; 57(3):345-9.

16. Tauchen ALO. A contribuição da odontologia do trabalho no programa de saúde ocupacional: verificando as condições de saúde bucal de trabalhadores de uma agroindústria do sul do Brasil [Dissertação de Mestrado]. São Paulo: Faculdade de Odontologia da Universidade de São Paulo; 2006.

17. Brasil. Ministério da Saúde. Projeto SB Brasil 2003: condições de saúde bucal da população brasileira 2002-2003. Resultados Principais. Brasília; 2004.

18. Pizzato E. A saúde bucal no contexto da saúde do trabalhador: análise dos modelos de atenção [Dissertação de Mestrado]. Araçatuba, SP: Faculdade de Odontologia da Universidade Estadual de São Paulo; 2002.

19. Tomita NE, Chinellato LEM, Lauris JRP, Kussano CM, Mendes HJ, Cardoso MTV. Oral health of building construction workers: an epidemiological approach. J Appl Oral Sci $2005 ; 13(1): 24-7$.

20. Almeida TF. Exposição ocupacional a névoas ácidas e doença periodontal [Dissertação de Mestrado]. Salvador: Faculdade de Odontologia da Universidade Federal da Bahia; 2005.

21. Brasil. Conselho Federal de Odontologia. Resolução no 25, de 16 de maio de 2002. Diário Oficial da União, Brasília, 28 de maio de 2002, seção 1, p. 148-9.

22. Lacerda JT, Ribeiro JD, Ribeiro DM, Traebert J. Prevalência da dor orofacial e seu impacto no desempenho diário em trabalhadores das indústrias têxteis do município de Laguna, SC. Cien Saude Colet 2011; 16(10):4275-82.

23. Mello PB. Odontologia do trabalho: uma revisão multidisciplinar. Rio de Janeiro: Livraria e Editora Rubio; 2006.

24. Mazzilli LEN. Odontologia do Trabalho. 2. ed. São Paulo: Livraria Santos; 2007.

25. Midorikawa ET. A odontologia em saúde do trabalhador como uma nova especialidade profissional: definição do campo de atuação do cirurgião-dentista na equipe de saúde do trabalhador [Dissertação de Mestrado]. São Paulo: Faculdade de Odontologia da Universidade de São Paulo; 2000.

26. Miller J. Waste of dental pain. Int Dent J 1978; 28(1):66-71.

27. Hooper HA. Dental services in industry: observations on their effects in the reduction on absenteeism. Industrial Medicine 1942; 11:157-62.

28. Almeida TF, Vianna MIP. O papel da epidemiologia no planejamento das ações de saúde bucal do trabalhador. Saúde Soc 2005; 14(3):144-54.

29. Duffy B. Dental problems in the offshore oil and gas industry: a review. Occup Med 1996; 46(1):79-83.

30. Peres AS, Olympio KPK, Cunha LSC, Bardal PAP. Odontologia do trabalho e sistema único de saúde - uma reflexão. Rev ABENO 2003; 4(1):38-41.

Endereço para correspondência:

Tatiana Frederico de Almeida

Rua João Bião de Cerqueira 251, Pituba

41830.580 Salvador, BA

Telefone: 7133514447 / 99334886

E-mail: tatifrederico@yahoo.com.br 